\section{Cartap poisoning: Additional thoughts}

Sir,

The article by Praveen Kumar et al..$^{[1]}$ is useful and timely, as practitioners and emergency physicians have started seeing cases of cartap poisoning. In response to the interesting report, ${ }^{[1]}$ we would like to share five more pieces of information with reference to its action, metabolism (P450), alterations in the level of lipoproteins, diagnosis and toxicity.

Indiscriminate use of huge organochlorine and organophosphate insecticides in the past have caused serious problems, like human health hazards, pest resurgence and threat to nontarget beneficial organisms, which has opened newer avenues in developing novel insecticides from plant and animal origin. Cartap, an analogue of nereistoxin, belongs to a relatively new class of insecticidal chemistry, and it acts directly and selectively at the noncompetitive inhibiting site: neuronal nicotinic acetylcholine receptor, producing inhibitory neurotoxicity. The toxicity of cartap gets enhanced while the patients concomitantly consume foods or drugs of P450 inhibitor. ${ }^{[2,3]}$ Hyperlipidemia was observed in patients with cartap poisoning, and it can act as a potential biomarker of toxicity in human beings. ${ }^{[4]}$

Cartap poisoning may likely be misdiagnosed as organophosphate or organochlorine poisoning because of several similarities in clinical presentation. ${ }^{[3]}$ Hence, practitioners and emergency physicians should be aware of this particular poisoning, as the management of these two are different. Despite the original belief that cartap has low mammalian toxicity, one would like to exercise caution in making such a statement, as it becomes toxic if the amount consumed is more than $75 \mathrm{~mL}$ of solution containing $50 \%$ cartap along with alcohol. The concentration of cartap in the formula is an important factor determining prognosis. ${ }^{[5]}$

As self-poisoning due to newer agents is an emerging problem, practitioners and emergency physicians face new challenges for want of prior human data on clinical features, biochemical abnormalities and case fatality, or if existing data are not effectively communicated to clinicians, medical educators or regulators. In view of the current status of cartap poisoning and potential toxicity in humans, a heightened awareness has to be made on this compound, clinical features, laboratory manifestations, therapeutic aspects and its toxicity among practitioners and students of health sciences.

\section{Acknowledgment}

The authors thank Dr. K. Arthanari, MS, for his logistic support.

\section{Subramanian Senthilkumaran, Namasivayam Balamurugan, Ritesh G. Menezes ${ }^{1}$, Ponniah Thirumalaikolundusubramanian ${ }^{2}$ \\ Sri Gokulam Hospitals and Research Institute, Salem, Tamil Nadu, 'Srinivas Institute of Medical Sciences and Research Centre, Mangalore, ${ }^{2}$ Chennai Medical College Hospital and Research Center, Irungalur, Trichy, India}

Correspondence: Dr. Subramanian Senthilkumaran, Department of Emergency and Critical Care Medicine, Sri Gokulam Hospital and Research Institute, Salem - 636 004, Tamil Nadu, India. E-mail: maniansenthil@yahoo.co.in

\section{References}

1. Praveen Kumar AS, Amalnath D, Dutta TK. Cartap poisoning: A rare case report. Indian J Crit Care Med 2011;15:233-5.

2. Lee SJ, Tomizawa M, Casida JE. Nereistoxin and cartap neurotoxicity attributable to direct block of the insect nicotinic receptor/channel. J Agric Food Chem 2003;51:2646-52

3. Senthilkumaran S, Balamurgan N, Thirumalaikolundusubramanian P. Emerging epidemic of cartap self-poisoning in Southern India: A prospective observational study. J Gen Med 2007;19:54-5.

4. Rai DK, Rai PK, Gupta A, Watal G, Sharma B. Cartap and carbofuran induced alterations in serum lipid profile of wistar rats. Indian J Clin Biochem 2009;24:198-201.

5. Kurisaki E, Kato N, Ishida T, Matsumoto A, Shinohara K, Hiraiwa K. Fatal human poisoning with Padan: A cartap-containing pesticide. Clin Toxicol 2010;48:153-5.

\begin{tabular}{|l|l|}
\hline \multicolumn{2}{|c|}{ Access this article online } \\
\hline Quick Response Code: & Website: \\
\hline
\end{tabular}

